\title{
Multilevel and SEM Approaches to Growth Curve Modeling
}

Joop Hox And Reinoud D. Stoel

Volume 3, pp. 1296-1305

in

Encyclopedia of Statistics in Behavioral Science

ISBN-13: 978-0-470-86080-9

ISBN-10: 0-470-86080-4

Editors

Brian S. Everitt \& David C. Howell

(C) John Wiley \& Sons, Ltd, Chichester, 2005 


\section{Multilevel and SEM Approaches to Growth Curve Modeling}

\section{Introduction}

A broad range of statistical methods exists for analyzing data from longitudinal designs (see Longitudinal Data Analysis). Each of these methods has specific features and the use of a particular method in a specific situation depends on such things as the type of research, the research question, and so on. The central concern of longitudinal research, however, revolves around the description of patterns of stability and change, and the explanation of how and why change does or does not take place [9].

A common design for longitudinal research in the social sciences is the panel or repeated measures design (see Repeated Measures Analysis of Variance), in which a sample of subjects is observed at more than one point in time. If all individuals provide measurements at the same set of occasions, we have a fixed occasions design. When occasions are varying, we have a set of measures taken at different points in time for different individuals. Such data occur, for instance, in growth studies, where individual measurements are collected for a sample of individuals at different occasions in their development (see Growth Curve Modeling). The data collection could be at fixed occasions, but the individuals have different ages. The distinction between fixed occasions designs and varying occasions designs is important, since they may lead to different analysis methods.

Several distinct statistical techniques are available for the analyses of panel data. In recent years, growth curve modeling has become popular [11, 12, 13, 24]. All subjects in a given population are assumed to have developmental curves of the same functional form (e.g., all linear), but the parameters describing their curves may differ. With linear developmental curves, for example, there may be individual differences in the initial level as well as in the growth rate or rate of change. Growth curve analysis is a statistical technique to estimate these parameters. Growth curve analysis is used to obtain a description of the mean growth in a population over a specific period of time (see Growth Curve Modeling). However, the main emphasis lies in explaining variability between subjects in the parameters that describe their growth curves, that is, in interindividual differences in intraindividual change [25].

The model on which growth curve analysis is based, the growth curve model, can be approached from several perspectives. On the one hand, the model can be constructed as a standard two-level multilevel regression (MLR) model [4, 5, 20] (see Linear Multilevel Models). The repeated measures are positioned at the lowest level (level-1 or the occasion level), and are then treated as nested within the individuals (level-2 or the individual level), the same way as a standard cross-sectional multilevel model treats children as being nested within classes. The model can therefore be estimated using standard MLR software. On the other hand, the model can be constructed as a structural equation model (SEM). Structural equation modeling uses latent variables to account for the relations between the observed variables, hence the name latent growth curve (LGC) model. The two approaches can be used to formulate equivalent models, providing identical estimates for a given data set [3].

\section{The Longitudinal Multilevel or Latent Growth Curve Model}

Both MLR and LGC incorporate the factor 'time' explicitly. Within the MLR framework time is modeled as an independent variable at the lowest level, the individual is defined at the second level, and explanatory variables can be included at all existing levels. The intercept and slope describe the mean growth. Interindividual differences in the parameters describing the growth curve are modeled as random effects for the intercept and slope of the time variable. The LGC approach adopts a latent variable view. Time is incorporated as specific constrained values for the factor loadings of the latent variable that represents the slope of the growth curve; all factor loadings of the latent variable that represents the intercept are constrained to the value of 1 . The latent variable means for the intercept and slope factor describe the mean growth. Interindividual differences in the parameters describing the growth curve are modeled as the (co)variances of the intercept and slope factors. The mean and covariance structure of the latent variables in LGC analysis correspond to the fixed and 


\section{Multilevel and SEM Approaches to Growth Curve Modeling}

random effects in MLR analysis, and this makes it possible to specify exactly the same model as a LGC or MLR model [23]. If this is done, exactly the same parameter estimates will emerge, as will be illustrated in the example.

The general growth curve model, for the repeatedly measured variable $y_{t i}$ of individual $i$ at occasion $t$, may be written as:

$$
\begin{aligned}
y_{t i} & =\lambda_{0 t} \eta_{0 i}+\lambda_{1 t} \eta_{1 i}+\gamma_{2 t} x_{t i}+\varepsilon_{t i} \\
\eta_{0 i} & =v_{0}+\gamma_{0} z_{i}+\zeta_{0 i} \\
\eta_{1 i} & =v_{1}+\gamma_{1} z_{i}+\zeta_{1 i},
\end{aligned}
$$

where $\lambda_{1 t}$ denotes the time of measurement and $\lambda_{0 t}$ a constant equal to the value of 1 . Note that in a fixed occasions design $\lambda_{1 t}$ will typically be a consecutive series of integers (e.g., $[0,1,2, \ldots, \mathrm{T}])$ equal to all individuals, while in a varying occasions design $\lambda_{1 t}$. can take on different values across individuals. The individual intercept and slope of the growth curve are represented by $\eta_{0 i}$ and $\eta_{1 i}$, respectively, with expectations $v_{0}$ and $v_{1}$, and random departures or residuals, $\zeta_{0 i}$ and $\zeta_{1 i}$, respectively. $\gamma_{2 t}$ represents the effect of the time-varying covariate $x_{t i} ; \gamma_{0}$ and $\gamma_{1}$ are the effects of the time-invariant covariate on the initial level and linear slope. Time-specific deviations are represented by the independent and identically standard normal distributed $\varepsilon_{t i}$, with variance $\sigma_{\varepsilon}^{2}$. The variances of $\zeta_{0 i}$ and $\zeta_{1 i}$, and their covariance are represented by:

$$
\Sigma_{\zeta}=\left[\begin{array}{cc}
\sigma_{0}^{2} & \\
\sigma_{01} & \sigma_{1}^{2}
\end{array}\right] .
$$

Furthermore, it is assumed that $\operatorname{cov}\left(\varepsilon_{i t}, \varepsilon_{i t^{\prime}}\right)=0$, $\operatorname{cov}\left(\varepsilon_{i t}, \eta_{i 0}\right)=0, \operatorname{cov}\left(\varepsilon_{i t}, \eta_{i 1}\right)=0$.

Within the longitudinal MLR model $\eta_{0 i}$ and $\eta_{1 i}$ are the random parameters, and $\lambda_{1 t}$ is an observed variable representing time. In the LGC model $\eta_{0 i}$ and $\eta_{1 i}$ are the latent variables and $\lambda_{0 t}$ and $\lambda_{1 t}$ are parameters, that is, factor loadings. Thus, the only difference between the models is the way time is incorporated in the model. In the MLR model time is introduced as a fixed explanatory variable, whereas in the LGC model it is introduced via the factor loadings. So, in the longitudinal MLR model an additional variable is added, and in the LGC model the factor loadings for the repeatedly measured variable are constrained in such a way that they represent time. The consequence of this is that with reference to the basic growth curve model, MLR is essentially a univariate approach, with time points treated as observations of the same variable, whereas the LGC model is essentially a multivariate approach, with each time point treated as a separate variable [23]. Figure 1 presents a path diagram depicting a LGC model for four measurement occasions, for simplicity without covariates. Following SEM conventions, the first path for the latent slope factor, which is constrained to equal zero, is usually not present in the diagram.

The specific ways MLR and LGC model 'time' have certain consequences for the analysis. In the LGC approach, $\lambda_{1 t}$ cannot vary between subjects, which makes it best suited for a fixed occasions design. LGC modeling can be used for designs with varying occasions by modeling all existing occasions and viewing the varying occasions as a missing data problem, but when the number of existing cases is large this approach becomes unmanageable. In the MLR approach, $\lambda_{1 t}$ is simply a time-varying explanatory variable that can take on any variable, which makes MLR the best approach if there are a large number of varying occasions. There are also some differences between the LGC and MLR approach in the ways the model can be extended. In the LGC approach, it is straightforward to embed the LGC model in a larger path model, for instance, by combining several growth curves in one model, or by using the intercept and slope factors as predictors for outcome values measured at a later occasion. The MLR approach does not deal well with such extended models. On the other hand, in the MLR approach it is simple to add more levels, for instance to model a growth process of pupils nested in classes nested in schools. In the LGC approach, it is possible to embed a LGC model in a two-level structural equation model [14], but adding more levels is problematic.

\section{Example}

We will illustrate the application of both the MLR model and the LGC model using a hypothetical study in which data on the language acquisition of 300 children were collected during primary school at 4 consecutive occasions. Besides this, data were collected on the children's intelligence, as well as, on each occasion a measure of their emotional well-being. The same data have been analyzed by Stoel, et al. [23], who also discuss extensions and applications of both models. 


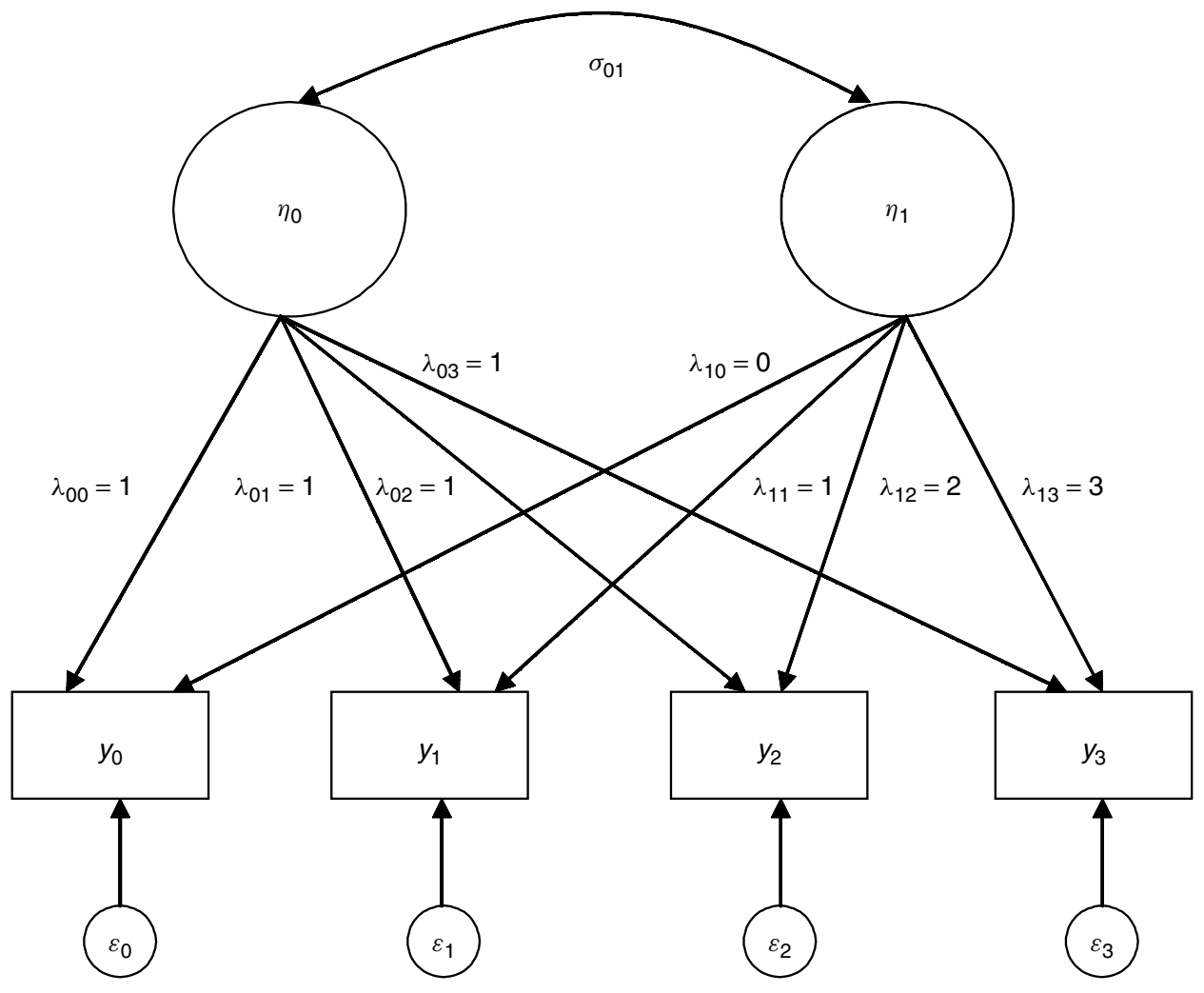

Figure 1 Path diagram of a four-wave latent growth curve model

The aim of the study is testing the hypothesis that there exists substantial growth in language acquisition, and that there is substantial variability between the children in their growth curves. Given interindividual differences in the growth curves, it is hypothesized that intelligence explains (part of) the interindividual variation in the growth curves and that emotional well-being explains the time-specific deviations from the mean growth curve. The covariance matrix and mean vector are presented in Table 1.

Analyzing these data using both the MLR and LGC model with Maximum Likelihood estimation leads to the parameter estimates presented in Table 2 .

Table 1 Covariance matrix and means vector

\begin{tabular}{|c|c|c|c|c|c|c|c|c|c|c|}
\hline & $\mathrm{y}_{1}$ & $\mathrm{y}_{2}$ & $\mathrm{y}_{3}$ & $\mathrm{y}_{4}$ & $\mathrm{x}_{1}$ & $\mathrm{x}_{2}$ & $\mathrm{x}_{3}$ & $\mathrm{X}_{4}$ & $\mathrm{z}$ & means \\
\hline $\mathrm{y}_{1}$ & 1.58 & & & & & & & & & 9.83 \\
\hline $\mathrm{y}_{2}$ & 1.28 & 4.15 & & & & & & & & 11.72 \\
\hline$y_{3}$ & 1.52 & 4.91 & 8.90 & & & & & & & 13.66 \\
\hline $\mathrm{y}_{4}$ & 1.77 & 6.83 & 11.26 & 17.50 & & & & & & 15.65 \\
\hline $\mathrm{x}_{1}$ & 0.99 & -0.08 & -0.26 & -0.33 & 2.17 & & & & & 0.00 \\
\hline $\mathrm{x}_{2}$ & 0.16 & 1.44 & 0.17 & 0.18 & 0.13 & 2.56 & & & & 0.00 \\
\hline$x_{3}$ & 0.07 & -0.13 & 1.2 & -0.27 & -0.06 & -0.09 & 2.35 & & & 0.00 \\
\hline $\mathrm{x}_{4}$ & 0.08 & 0.23 & 0.46 & 1.98 & 0.03 & -0.04 & 0.10 & 2.24 & & 0.00 \\
\hline $\mathrm{z}$ & 0.34 & 1.28 & 2.01 & 3.01 & -0.08 & 0.07 & 0.01 & 0.06 & 0.96 & 0.00 \\
\hline
\end{tabular}

Note: $\mathrm{y}=$ language acquisition, $\mathrm{x}=$ emotional well-being, $\mathrm{z}=$ intelligence. 
Table 2 Maximum likelihood estimates of the parameters of (1), using multilevel regression and latent growth curve analysis

\begin{tabular}{lcc}
\hline \multicolumn{1}{c}{ Parameter } & MLR & LGC \\
\hline Fixed part & $9.89(.06)$ & $9.89(.06)$ \\
$\nu_{0}$ & $1.96(.05)$ & $1.96(.05)$ \\
$\nu_{1}$ & $0.40(.06)$ & $0.40(.06)$ \\
$\gamma_{0}$ & $0.90(.05)$ & $0.90(.05)$ \\
$\gamma_{1}$ & $0.55(.01)$ & $0.55(.01)$ \\
$\gamma_{2}$ & & \\
Random part & $0.25(.01)$ & $0.25(.01)$ \\
$\sigma_{\varepsilon}^{2}$ & $0.78(.08)$ & $0.78(.08)$ \\
$\sigma_{0}^{2}$ & $0.64(.06)$ & $0.64(.06)$ \\
$\sigma_{1}^{2}$ & $0.00(.05)$ & $0.00(.05)$ \\
$\sigma_{01}$ &
\end{tabular}

Note: Standard errors are given in parentheses. The Chi-square test of model fit for the LGC model: $\chi^{2}(25)=37.84(p=.95)$; RMSEA $=0.00$. For the MLR model: $-2 * \log$ likelihood $=$ 3346.114.

The first column of Table 2 presents the relevant parameters; the second and third columns show the parameter estimates of respectively the MLR, and LGC model.

As one can see in Table 2 the parameter estimates are the same and, consequently, both approaches would lead to the same substantive conclusions. According to the overall measure of fit provided by SEM, the model seems to fit the data quite well. Thus, the conclusions can be summarized as follows. After controlling for the effect of the covariates, a mean growth curve emerges with an initial level of 9.89 and a growth rate of 1.96. The significant variation between the subjects around these mean values implies that subjects start their growth process at different values and grow subsequently at different rates. The correlation between initial level and growth rate is zero. In other words, the initial level has no predictive value for the growth rate. Intelligence has a positive effect on both the initial level and growth rate, leading to the conclusion that children who are more intelligent show a higher score at the first measurement occasion and a greater increase in language acquisition than children with lower intelligence. Emotional well-being explains the timespecific deviations from the mean growth curve. That is, children with a higher emotional wellbeing at a specific time point show a higher score on language acquisition than is predicted by their growth curve.

\section{Dichotomous and Ordinal Data}

Both conventional structural equation modeling and multilevel regression analysis assume that the outcome variable(s) are continuous and have a (multivariate) normal distribution. In practice, many variables are measured as ordinal categorical variables, for example, the responses on a five- or sevenpoint Likert attitude question. Often, researchers treat such variables as if they were continuous and normal variables. If the number of response categories is fairly large and the response distribution is symmetric, treating these variables as continuous normal variable appears to work quite well. For instance, Bollen and Barb [2] show in a simulation study that if bivariate normal variables are categorized into at least five response categories, the differences between the correlation between the original variables and the correlation of the categorized variables is small (see Categorizing Data). Johnson and Creech [7] show that this also holds for parameter estimates and model fit. However, when the number of categories is smaller than five, the distortion becomes sizable. It is clear that such variables require special treatment.

A Categorical ordinal variable can be viewed as a crude observation of an underlying latent variable. The same model that is used for the continuous variables is used, but it is assumed to hold for the underlying latent response. The residuals are assumed to have a standard normal distribution, or a logistic distribution. The categories of the ordinal variable arise from applying thresholds to the latent continuous variable. Assume that we have an ordered categorical variable with three categories, for example, 'disagree', 'neutral', and 'agree.' The relation of this variable to the underlying normal latent variable is depicted in Figure 2.

The position on the latent variable determines which categorical response is observed. Specifically,

$$
y_{i}=\left\{\begin{array}{lll}
1, & \text { if } \quad y_{i}^{*} \leq \tau_{1} \\
2, & \text { if } \quad \tau_{1}<y_{i}^{*} \leq \tau_{2} \\
3, & \text { if } \quad \tau_{2}<y_{i}^{*},
\end{array}\right.
$$

where $y_{i}$ is the observed categorical variable, $y_{i}^{*}$ is the latent continuous variable, and $\tau_{1}$ and $\tau_{2}$ are the thresholds. Note that a dichotomous variable only has one threshold, which becomes the intercept in a regression equation.

To analyze ordered categorical data in a multilevel regression context, the common approach is 


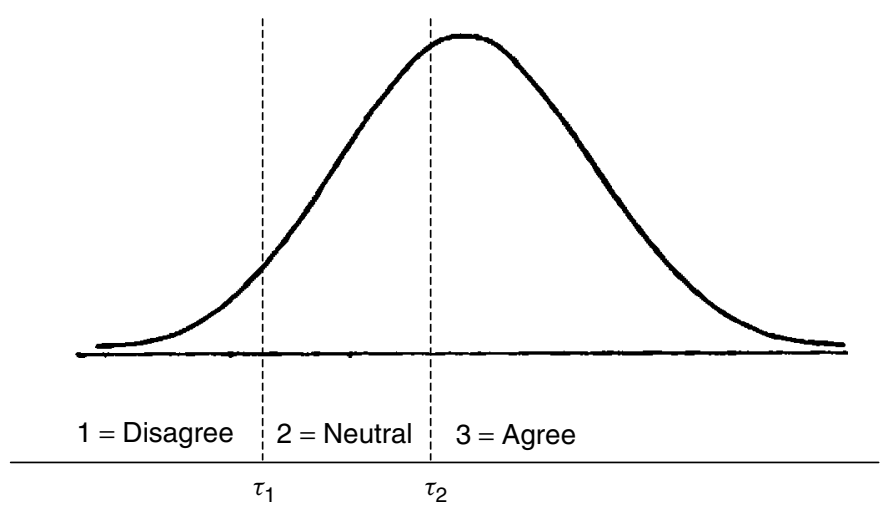

Figure 2 Illustration of two thresholds underlying a three-category variable

to assume a normal or a logistic distribution (see Catalogue of Probability Density Functions). There are several estimation methods available; most software relies on a Taylor series approximation to the likelihood, although some software is capable of numerical integration of the likelihood. For a discussion of estimation in nonnormal multilevel models computational details, we refer to the literature, for example $[4,20]$. The common approach in structural equation modeling is to estimate polychoric correlations, that is, the correlations between the underlying latent responses, and apply conventional SEM methods. This approach also assumes a standard normal distribution for the residuals. For statistical issues and computational details, we refer to the literature, for example [1, 8] (see Structural Equation Modeling: Categorical Variables). The important point here for both types of modeling is that when the number of categories of an outcome variable is small, using an analysis approach that assumes continuous variables may lead to strongly biased results.
To give an indication of the extent of the bias, the outcome variables of the language acquisition example were dichotomized on their common median. This leads to a data set where the number of 'zero' and 'one' scores on the four Y variables taken together is $50 \%$ each, with the proportion of 'one' scores goes up from 0.04 through 0.44 at the first occasion to 0.72 to 0.81 at the last occasion. Table 3 presents the results of three multilevel analyses for a model with a linear random effect for time: (1) Maximum likelihood estimation on a standardized continuous outcome $Z$ (mean zero and variance one across Y1 to Y4) assuming normality, (2) Maximum Likelihood estimation on the dichotomized outcome $D$ assuming normality, and (3) approximate Maximum Likelihood estimation on the dichotomized outcome $D$ assuming a logistic model and using a Laplace approximation (Laplace6 in HLM5, [22]).

The parameter estimates are different across all three methods. This is not surprising, because the $Z$ scores are standardized to a mean of 0 and a variance of 1 , the dichotomous variables have an overall mean

Table 3 Estimates of the parameters of (1), using multilevel regression and different estimation methods

\begin{tabular}{lccc}
\hline Parameter & ML on $Z$ assuming normality & ML on $D$ assuming normality & Approximate $\mathrm{ML}$ on $D$ assuming logistic \\
\hline Fixed Part & -0.82 & 0.11 & -2.44 \\
$\nu_{0}$ & 0.54 & 0.26 & 1.80 \\
$v_{1}$ & & & \\
Random part & 0.07 & 0.10 & - \\
$\sigma_{\varepsilon}^{2}$ & 0.07 & 0.01 & 0.24 \\
$\sigma_{0}^{2}$ & 0.12 & 0.01 & 0.60 \\
$\sigma_{1}^{2}$ & 0.03 & 0.01 & 0.38 \\
$\sigma_{01}$ & &
\end{tabular}


of 0.5 and a variance of 0.25 , and the underlying continuous variable $y^{*}$ has a mean of 0 and a residual variance of approximately 3.29. However, statistical tests on the variance components lead to drastically different conclusions. An ordinary likelihood-ratio test on the variances is not appropriate, because the null-hypothesis is a value that lies on the boundary of the parameter space. Instead, we apply the chi-square test described by Raudenbush and Bryk [21], which is based on the residuals. The multilevel regression analysis on the continuous $Z$-scores leads to significant variance components $(p<0.01)$. The multilevel logistic regression analysis on the dichotomized $D$ scores leads to variance components that are also significant $(p<0.01)$. On the other hand, the multilevel regression analysis on the dichotomized $D$-scores using standard Maximum Likelihood estimation for continuous outcomes, leads to variance components that are not significant $(p>0.80)$. Thus, for our example data, using standard Maximum Likelihood estimation assuming a continuous outcome on the dichotomized variable leads to substantively different and in fact misleading conclusions.

\section{Extensions}

The model in (1) can be extended in a number of ways. We will describe some of these extensions in this section separately, but they can in fact be combined in one model.

\section{Extending the Number of Levels}

First, let us assume that we have collected data on several occasions from individuals within classes, and that there are (systematic) differences between classes in intercept and slope. The model in (1) can easily account for such a 'three-level' structure by adding the class-specific subscript $j$. The model then becomes:

$$
\begin{aligned}
y_{t i j} & =\lambda_{0 t} \eta_{0 i j}+\lambda_{1 t} \eta_{1 i j}+\gamma_{2 t} x_{t i j}+\varepsilon_{t i j} \\
\eta_{0 i j} & =v_{0 j}+\gamma_{0} z_{i}+\zeta_{0 i j} \\
\eta_{1 i j} & =v_{1 j}+\gamma_{1} z_{i}+\zeta_{1 i j} \\
v_{0 j} & =v_{0}+\zeta_{2 j} \\
v_{1 j} & =v_{1}+\zeta_{3 j}
\end{aligned}
$$

and reflects the fact that the mean intercept and slope ( $v_{0 j}$ and $v_{0 j}$, respectively) may be different across classes. Note that (4) turns into (1) if $\zeta_{2 j}$ and $\zeta_{3 j}$ are constrained to zero. Incorporating classlevel covariates and additional higher levels in the hierarchy are straightforward.

\section{Extending the Measurement Model}

Secondly, the model in (1) can be easily extended to include multiple indicators of a construct at each occasion explicitly. Essentially this extension merges the growth curve model with a measurement (latent factor) model at each occasion. For example if $y_{t i}$ represented a mean score over $R$ items, we may recognize that $y_{t i}=\sum_{r=1}^{R} y_{r t i} / m$. Instead of modeling observed item parcels, the $R$ individual items $y_{r t i}$ can be modeled directly. That is, to model them, explicitly, as indicators of a latent construct or factor at each measurement occasion. A growth model may then be constructed to explain the variance and covariance among the first-order latent factors. This approach has been termed second-order growth modeling in contrast to first-order growth modeling on the observed indicators. Different names for the same model are 'curve-of-factors model' and 'multiple indicator latent growth model' [12]. Note that modeling multiple indicators in a longitudinal setting requires a test on the structure of the measurements, that is a test of measurement invariance or factorial invariance $[1,8]$. The model incorporating all $y_{r t i}$ explicitly then becomes:

$$
\begin{aligned}
y_{r t i} & =\alpha_{r}+\lambda_{r} \eta_{t i}+\varepsilon_{r i} \\
\eta_{t i} & =\lambda_{0 t} \eta_{0 i}+\lambda_{1 t} \eta_{1 i}+\gamma_{2 t} x_{t i}+\zeta_{t i} \\
\eta_{0 i} & =v_{0}+\gamma_{0} z_{i}+\zeta_{0 i} \\
\eta_{1 i} & =v_{1}+\gamma_{1} z_{i}+\zeta_{1 i},
\end{aligned}
$$

where $\alpha_{r}$ and $\lambda_{r}$ represent, respectively, the itemspecific intercept and factor loading of item $r$, and $\varepsilon_{r i}$ is a residual. $\eta_{t i}$ is an individual and time-specific latent factor corresponding to $y_{t i}$ of Model (1), $\zeta_{t i}$ is a random deviation corresponding to $\varepsilon_{t i}$ of Model (1). The growth curve model is subsequently built on the latent factor scores $\eta_{t i}$ with $\lambda_{1 t}$ representing the time of measurement and $\lambda_{1 t}$ a constant equal to the value of 1 . This model thus allows for a separation of measurement error $\varepsilon_{r i}$ and individual time-specific 
deviation $\zeta_{t i}$. In Model (1) these components are confounded in $\varepsilon_{t i}$.

\section{Nonlinear Growth}

The model discussed so far assumes linear growth. The factor time is incorporated explicitly in the model by constraining $\lambda_{1 t}$ in (1) explicitly to known values to represent the occasions at which the subjects ere measured. This is, however, not a necessary restriction; it is possible to estimate a more general, that is nonlinear, model in which values of $\lambda_{1 t}$ are estimated (see Nonlinear Mixed Effects Models; Nonlinear Models). Thus, instead of constraining $\lambda_{1 t}$ to, for example $[0,1,2,3 \ldots T]$, some elements are left free to be estimated, providing information on the shape of the growth curve. For purposes of identification, at least two elements of $\lambda_{1 t}$ need to be fixed. The remaining values are then estimated to provide information on the shape of the curve; $\lambda_{1 t}$ then becomes $\left[0,1, \lambda_{12}, \lambda_{13}, \ldots, \lambda_{1 T-1}\right]$. So, essentially, a linear model is estimated, while the nonlinear interpretation comes from relating the estimated $\lambda_{1 t}$ to the real time frame $[13,24]$. The transformation of $\lambda_{1 t}$ to the real time frame gives the nonlinear interpretation.

\section{Further Extensions}

Further noteworthy extensions of the standard growth model in (1) which we will briefly sum up here are:

- The assumption of independent and identically distributed residuals can be relaxed. In other words, the model in (1) may incorporate a more complex type of residual structure. In fact, any type of residual structure can be implemented, provided the resulting model is identified.

- As stated earlier, the assumption that all individuals have been measured at the same measurement occasions as implied by Model (1) can be relaxed by giving $\lambda_{1 t}$ in (1) an individual subscript $i . \lambda_{1 t i}$ can subsequently be partly, or even completely different across individuals. However, using LGC modeling requires that we treat different $\lambda_{1 t i}$ 's across subjects as a balanced design with missing data (i.e., that not all subjects have been measured at all occasions), and assumptions about the missing data mechanism need to be made.
- Growth mixture modeling provides an interesting extension of conventional growth curve analysis. By incorporating a categorical latent variable it is possible to represent a mixture of subpopulations where population membership is not known but instead must be inferred from the data $[15,16$, 18]. See Li et al. [10], for a didactic example of this methodology.

\section{Estimation and Software}

When applied to longitudinal data as described above, the MLR and LGC model are identical; they only differ in their representation. However, these models come from different traditions, and the software was originally developed to analyze different problems. This has consequences for the way the data are entered into the program, the choices the analyst must make, and the ease with which specific extensions of the model are handled by the software.

LGC modeling is a special case of the general approach known as structural equation modeling (SEM). Structural equation modeling is inherently a multivariate analyst method, and it is therefore straightforward to extend the basic model with other (latent or observed) variables. Standard SEM software abounds with options to test the fit of the model, compare groups, and constrain parameters within and across groups. This makes SEM a very flexible analysis tool, and LGC modeling using SEM shows this flexibility. Typically, the different measurement occasions are introduced as separate variables. Time-varying covariates are also introduced as separate variables that affect the outcome measures at the corresponding measurement occasions. Time invariant covariates are typically incorporated in the model by giving these an effect on the latent variables that represent the intercept or the slope. However, it is also possible to allow the time invariant covariates to have direct effects on the outcome variables at each measurement occasion. This leads to a different model. In LGC modeling using SEM, it is a straightforward extension to model effects of the latent intercept and slope variables on other variables, including analyzing two LGC trajectories in one model and investigating how their intercepts and slopes are related.

The flexibility of LGC analysis using SEM implies that the analyst is responsible for ensuring that the 
model is set up properly. For instance, one extension of the basic LGC model discussed in the previous section is to use a number of indicators for the outcome measure and extend the model by including a measurement model. In this situation, the growth model is modeled on the consecutive latent variables. To ensure that the measurement model is invariant over time, the corresponding factor loadings for measurements across time must be constrained to be equal. In addition, since the LGC model involves changes in individual scores and the overall mean across time, means and intercepts are included in the model, and the corresponding intercepts must also be constrained equal over time.

Adding additional levels to the model is relatively difficult using the SEM approach. Muthén [14] has proposed a limited information Maximum Likelihood method to estimate parameters in two-level SEM. This approach works well [6], and can be implemented in standard SEM software [5]. Since the LGC model can be estimated using standard SEM, two-level SEM can include a LGC model at the individual (lowest) level, with groups at the second level. However, adding more levels is cumbersome in the SEM approach.

Multilevel Regression (MLR) is a univariate method, where adding an extra (lowest) level for the variables allows analysts to carry out multivariate analyses. So, growth curve analysis using MLR is accomplished by adding a level for the repeated measurement occasions. Most MLR software requires that the data matrix is organized by having a separate row for each measurement occasion within each individual, with the time invariant individual characteristics repeated for occasions within the same individual. Adding time-varying or time invariant covariates to the model is straightforward; they are just added as predictor variables. Allowing the time invariant covariates to have direct effects on the outcome variables at each measurement occasion is more complicated, because in the MLR approach this requires specifying interactions of these predictors with dummy variables that indicate the measurement occasions.

Adding additional levels is simple in MLR; after all, this is what the software was designed for. The maximum number of levels is defined by software restrictions; the current record is MLwiN [20], which is designed to handle up to 50 levels. This may seem excessive, but many special analyses are set up in multilevel regression software by including an extra level. This is used, for instance, to model multivariate outcomes, cross-classified data, and specify measurement models. For such models, a nesting structure of up to five levels is not unusual, and not all multilevel regression software can accommodate this.

The MLR model is more limited than SEM. For instance, it is not possible to let the intercept and slopes act as predictors in a more elaborate path model. The limitations show especially when models are estimated that include latent variables. For instance, models with latent variables over time that are indicated by observed variables, easy to specify in SEM, can be set up in MLR using an extra variable level. At this (lowest) level, dummy variables are used to indicate variables that belong to the same construct at different measurement occasions. The regression coefficients for these dummies are allowed to vary at the occasion level, and they are interpreted as latent variables in a measurement model. However, this measurement model is more restricted than the measurement in the analogous SEM. In the MLR approach, the measurement model is a factor model with equal loadings for all variables, and one common error variance for the unique factors. In some situations, for instance, when the indicators are items measured using the same response scale, this restriction may be reasonable. It also ensures that the measurement model is invariant over time. The important issue is of course that this restriction cannot be relaxed in the MLR model, and it cannot be tested.

Most modern structural equation modeling software can be used to analyze LGC models. If the data are unbalanced, either by design or because of panel attrition, it is important that the software supports analyzing incomplete data using the raw Likelihood. If there are categorical response variables, it is important that the software supports their analysis. At the time of writing, only Muthén's software Mplus supports the combination of categorical incomplete data [17].

Longitudinal data can be handled by all multilevel software. Some software supports analyzing specific covariance structures over time, such as autoregressive models. When outcome variables may be categorical, there is considerable variation in the estimation methods employed. Most multilevel regression relies on Taylor series linearization, but increasingly numerical integration is used, which is regarded as more accurate. 
A recent development in the field is that the distinction between MLR and LGC analysis is blurring. Advanced structural equation modeling software is now incorporating some multilevel features. Mplus, for example, goes a long way towards bridging the gap between the two approaches $[15,16]$. On the other hand MLR software is incorporating features of LGC modeling. Two MLR software packages allow linear relations between the growth parameters: HLM [22] and GLLAMM [19]. HLM offers a variety of residual covariance structures for MLR models. The GLLAMM framework is especially powerful; it can be viewed as a multilevel regression approach that allows factor loadings, variable-specific unique variances, as well as structural equations among latent variables (both factors and random coefficients). In addition, it supports categorical and incomplete data. As the result of further developments in both statistical models and software, the two approaches to growth curve modeling may in time merge (see Software for Statistical Analyses; Structural Equation Modeling: Software).

\section{Discussion}

Many methods are available for the analysis of longitudinal data. There is no single preferred procedure, since different substantial questions dictate different data structures and statistical models. This entry focuses on growth curve analysis. Growth curve analysis, and its SEM variant latent growth curve analysis, has advantages for the study of change if it can be assumed that change is systematically related to the passage of time. Identifying individual differences in change, as well as understanding the process of change are considered critical issues in developmental research by many scholars. Growth curve analysis explicitly reflects on both intra-individual change and interindividual differences in such change.

In this entry, we described the general growth curve model and discussed differences between the multilevel regression approach and latent growth curve analysis using structural equation modeling. The basic growth curve model has a similar representation, and gives equivalent results in both approaches. Differences exist in the ways the model can be extended. In many instances, latent growth curve analysis is preferred because of its greater flexibility. Multilevel Regression is preferable if the growth model must be embedded in a larger number of hierarchical data levels.

\section{References}

[1] Bollen, K.A. (1989). Structural Equations with Latent Variables., Wiley, New York.

[2] Bollen, K.A. \& Barb, K. (1981). Pearson's R and coarsely categorized measures, American Sociological Review 46, 232-239.

[3] Curran, P. (2003). Have multilevel models been structural equation models all along? Multivariate Behavior Research 38(4), 529-569.

[4] Goldstein, H. (2003). Multilevel Statistical Models, Edward Arnold, London.

[5] Hox, J.J. (2002). Multilevel Analysis: Techniques and Applications, Lawrence Erlbaum, Mahwah.

[6] Hox, J.J. \& Maas, C.J.M. (2001). The accuracy of multilevel structural equation modeling with pseudobalanced groups and small samples, Structural Equation Modeling 8, 157-174.

[7] Johnson, D.R. \& Creech, J.C. (1983). Ordinal measures in multiple indicator models: a simulation study of categorization error, American Sociological Review 48, 398-407.

[8] Kaplan, D. (2000). Structural Equation Modeling, Sage Publications, Thousand Oaks.

[9] Kessler, R.C. \& Greenberg, D.F. (1981). Linear Panel Analysis, Academic Press, New York.

[10] Li, F., Duncan, T.E., Duncan, S.C. \& Acock, A. (2001). Latent growth modeling of longitudinal data: a finite growth mixture modeling approach, Structural Equation Modeling 8, 493-530.

[11] McArdle, J.J. (1986). Latent variable growth within behavior genetic models, Behavior Genetics 16, 163-200.

[12] McArdle, J.J. (1988). Dynamic but structural equation modeling of repeated measures data, in Handbook of Multivariate Experimental Psychology, 2nd Edition, R.B. Cattel \& J. Nesselroade, eds, Plenum Press, New York, pp. 561-614.

[13] Meredith, W.M. \& Tisak, J. (1990). Latent curve analysis, Psychometrika 55, 107-122.

[14] Muthén, B. (1994). Multilevel covariance structure analysis, Sociological Methods \& Research 22, 376-398.

[15] Muthén, B. (2001). Second-generation structural equation modeling with a combination of categorical and continuous latent variables: New opportunities for latent class-latent growth modeling, in New Methods for the Analysis of Change, L.M. Collins \& A.G. Sayer, eds, American Psychological Association, Washington, pp. 291-322.

[16] Muthén, B.O. (2002). Beyond SEM: general latent variable modeling, Behaviormetrika 29, 81-117.

[17] Muthén, L.K. \& Muthén, B.O. (2004). Mplus Users Guide, Muthén \& Muthén, Los Angeles. 
[18] Muthén, B. \& Shedden, K. (1999). Finite mixture modeling with mixture outcomes using the EM algorithm, Biometrics 55, 463-469.

[19] Rabe-Hesketh, S., Skrondal, A. \& Pickles, A. (2004). Generalized multilevel structural equation modelling, Psychometrika 69, 167-190.

[20] Rasbash, J., Browne, W., Goldstein, H., Yang, M., Plewis, I., Healy, M., Woodhouse, G., Draper, D., Langford, I. \& Lewis, T. (2000). A User's Guide to MlwiN, Multilevel Models Project, University of London, London.

[21] Raudenbush, S.W. \& Bryk, A.S. (2002). Hierarchical Linear Models: Applications and Data Analysis Methods, Sage Publications, Newbury Park.

[22] Raudenbush, S., Bryk, A., Cheong, Y.F. \& Congdon, R. (2000). HLM 5. Hierarchical Linear and Nonlinear Modeling, Scientific Software International, Chicago.

[23] Stoel, R.D., Van den Wittenboer, G. \& Hox, J.J. (2003). Analyzing longitudinal data using multilevel regression and latent growth curve analysis, Metodologia de las Ciencas del Comportamiento 5, 21-42.

[24] Stoel, R.D., van den Wittenboer, G. \& Hox, J.J. (2004). Methodological issues in the application of the latent growth curve model, in Recent Developments in Structural Equation Modeling: Theory and Applications, K. van Montfort, H. Oud \& A. Satorra, eds, Kluwer Academic Publishers, Amsterdam, pp. 241-262.

[25] Willett, J.B. \& Sayer, A.G. (1994). Using covariance structure analysis to detect correlates and predictors of individual change over time, Psychological Bulletin 116, $363-381$.

(See also Structural Equation Modeling: Multilevel)

JoOP Hox AND ReINOUd D. Stoel 